Voix et Images

voixetimages

\title{
Entretien avec Monique LaRue
}

Jean-François Chassay et Katherine Roberts

Volume 28, numéro 2 (83), hiver 2003

Monique LaRue

URI : https://id.erudit.org/iderudit/006593ar

DOI : https://doi.org/10.7202/006593ar

Aller au sommaire du numéro

Éditeur(s)

Université du Québec à Montréal

\section{ISSN}

0318-9201 (imprimé)

1705-933X (numérique)

Découvrir la revue

\section{Citer ce document}

Chassay, J.-F. \& Roberts, K. (2003). Entretien avec Monique LaRue. Voix et Images, 28(2), 13-29. https://doi.org/10.7202/006593ar d'utilisation que vous pouvez consulter en ligne.

https://apropos.erudit.org/fr/usagers/politique-dutilisation/ 


\title{
Entretien avec Monique LaRue
}

\author{
J ean-François Chassay, Université du Québec à Montréal \\ Katherine Roberts, Bowling Green State University
}

V. et I. : À bien y penser, ce n'est pas tellement courant de voir publier ici au Québec des «romans d'idées», des romans, qui sans être didactiques, reposent sur de solides réflexions intellectuelles. Cette tendance est-elle liée à votre formation en philosophie? Comment s'est effectué ce passage de la philosophie à la littérature?

M. L.: D'une certaine manière, il me semble que ce que recherchent la philosophie et la littérature est très semblable : on pourrait ramener cela à une quête de sens. Je me rappelle avoir choisi la philosophie parce que c'était une démarche rigoureuse et que, par comparaison, les lettres m’apparaissaient comme un secteur «mou ». Ce qu'on m'avait jusque-là demandé d'accomplir en lettres m'était toujours apparu trop simple. Je trouvais que l'on pouvait dire n'importe quoi en littérature, je n'avais pas eu un bon enseignement. Au contraire, la philosophie m'apparaissait comme un domaine où justement, pour affronter le chaos qu'est la vie, on pouvait réfléchir de façon systématique, rationnelle et rigoureuse. C'était probablement à mes yeux la recherche d'une certaine sécurité à travers la raison. Parallèlement à ces études dites sérieuses, j'ai toujours lu de la fiction et j'ai eu très tôt le désir d'écrire, mais je ne faisais pas le lien entre les deux disciplines. J'ai donc fait un baccalauréat et une maîtrise en philosophie avant d'être confrontée à des difficultés de taille. Premièrement, le monde de la philosophie, je m'en suis vite aperçue, est un monde d'hommes comme on l'imagine peu. Il m'est apparu peu à peu qu'en philosophie, la femme est associée à tout ce qui est irrationnel. Bien sûr, il y a des exceptions comme Simone de Beauvoir qui fut certainement un phare pour moi, mais sinon, ce monde masculin m'était très difficile à supporter étant donné que, plus j’avançais en âge, plus les questions féministes devenaient importantes dans la société, et pour moi. Deuxièmement, l'abstraction philosophique m'a menée à une impasse totale après mon mémoire de maîtrise.

V. et I. : Q uel en était le sujet?

M. L. : La conception du langage chez Heidegger, notamment dans L'Être et le temps. 
V. et I. : C'est amusant de voir que la prédominance de la raison qui vous a fait opter pour la philosophie est, en quelque sorte, battue en brèche plus tard dans votre parcours par ce mémoire sur Heidegger dont la philosophie est un combat contre la raison.

M. L. : Oui, en effet, j'avançais vers quelque chose de plus littéraire. Je m'intéressais de plus en plus à la phénoménologie, en somme à ce qui était plus littéraire, plus de l'ordre de l'intuition. Après ce mémoire sur la refondation du langage, pour lequel j'avais essayé d'apprendre l'allemand, les études en philosophie devenaient trop abstraites pour que je persévère dans cette voie. Mais j'étais à ce moment-là à Paris pour la maîtrise, et Roland Barthes prenait des élèves étrangers. C'était bien pour moi, cette époque-là, parce que c'était celle de la sémiologie, où l'on voulait justement introduire en littérature une certaine rationalité, des méthodes venues de la science. Je suis donc allée travailler avec lui. Ce qui ne voulait pas dire grand-chose, sinon que de s'asseoir autour d'une table et d'écouter Barthes, car, comme la plupart des professeurs français, c'est bien connu, il ne s'occupait pas beaucoup de ses étudiants. Mais c'était un homme sympathique grâce à qui je me suis dirigée vers la littérature. Cela dit, il m'est resté beaucoup de ma formation philosophique; je m'en suis beaucoup servie parce que dès que l'on devient écrivain, la société nous demande de réfléchir; par conséquent, j'ai toujours continué à lire de la philosophie et cela marque mes romans. De même, je n'ai jamais lu autant de romans policiers, tant européens qu'américains, que pendant mes études en philosophie. Mais je n'écrivais pas de fiction à ce momentlà. Le passage vers la littérature s'est donc amorcé avec Roland Barthes lorsque j'ai délimité mon sujet de thèse. J'ai choisi de travailler sur un corpus de littérature pour enfants. J'ai procédé à une analyse discursive de cette littérature; j'essayais d'y discerner ce qui était purement pédagogique et didactique de ce qui pouvait relever du littéraire. Les spécificités de la littérarité étaient ce que je cherchais à définir. Avec le recul, il m’apparait évident qu'en sourdine je me dirigeais déjà vers la question de la maternité et de l'enfance, abordées concrètement dans La cohorte fictive.

V. et I. : Si la philosophie n'est pas à l'origine de l'écriture fictionnelle, est-ce que la maternité pourrait l'être, puisque dans La cohorte fictive on retrouve justement cette thématique, à travers une mise en scène de la naissance, mais aussi à un niveau métaphorique, dans celle de la gestation du texte lui-même? Y a-t-il une part d'autobiographie dans le discours de la narratrice qui écrit pendant que le bébé dort?

M. L. : Oui. Au départ, la situation est très personnelle. D'ailleurs, cela vient d'être repris par Marie Darrieussecq dans un roman qui s'appelle Le bébé, ce qui m’a rappelé cette période-là. Pour moi, comme je l'ai écrit dans ce roman, ces deux expériences - la création et la maternité étaient liées de très près. C'était aussi une réaction à ce monde extrême- 
ment masculin que j'avais fréquenté, où les hommes aiment bien parler d'accouchements intellectuels. Par ailleurs, l'expérience de la maternité, de l'accouchement, avait été peu traitée à l'époque du féminisme: c'était ma réponse à une certaine perception de la femme-mère par ce mouvement. Simone de Beauvoir, par exemple, a eu des propos durs envers la maternité. On disait en substance que l'émancipation de la femme devait passer par la libération de cette tâche biologique.

V. et I. : Cette mentalité était-elle aussi présente dans le mouvement féministe québécois à l'époque?

M. L. : Oh oui, c'était omniprésent. Faire un enfant à cette époque-là était tout simplement mal vu, cela se sentait très bien. C'était une décision qui prenait immédiatement une valeur idéologique. Dans les années 1980, avoir un troisième enfant (ce qui a été mon cas) paraissait très exagéré. Mais cela appelle à questionner la puissance des idéologies sur l'individu et sur la société en général. Je pense que la position de l'écrivain est de les suspecter, de les remettre en cause tout le temps. C'est une des fonctions du roman; tâche très difficile d'ailleurs à accomplir parce que, dans un très petit milieu comme le nôtre, on doit avoir du cran pour le faire.

V. et I. : Ce rapport au féminisme semble finalement assez conflictuel: il y avait un intérêt intellectuel indéniable, mais en même temps certaines idées chères au mouvement vous paraissaient inacceptables.

M. L.: C'est-à-dire que je suis évidemment féministe, ai-je besoin de le répéter, il est impossible de ne pas l'être. Cela me semble une évidence, mais malheureusement il faut toujours le dire. Est-ce qu'on peut être antiféministe? Non. Mais tout mouvement, toute idéologie finit par créer des conformismes, et assez rapidement. Or, la sensibilité de l'écrivain réside dans une allergie radicale à toute forme de dogmatisme, et consiste à détecter et à questionner les discours, tous les discours, ce qui m’a menée à me demander, par exemple, pourquoi le mouvement féministe rejetterait la moitié des femmes dans le monde ou à tout le moins celles qui ont décidé d'avoir des enfants. Le fait qu'un enfant se fasse avec un homme était peut-être en cause. C'est sûr que l'on peut avoir des enfants de bien d'autres manières, de plus en plus - je n'ai absolument rien contre - , mais en général c'est quand même cela qui se passe... Cette évidence devait être dite, cela peut paraître invraisemblable mais elle ne figurait pas nécessairement dans la correction politique de l'époque. Ensuite, à force de réflexion, il me semble que le féminisme s'est justement ouvert, le mouvement est devenu moins dur, moins dogmatique, et heureusement.

V. et I. : C'est peut-être aussi qu'à cette époque-là, à la fin des années 1970, le féminisme n'était pas le seul courant à être profondément dogmatique. Il y avait aussi beaucoup d'autres dogmatismes, c'était dans l'esprit du temps... 
M. L : Oui, absolument, et il y en a encore. Au Québec, cela arrive assez facilement que l'on devienne insensiblement dogmatique, ou du moins que les idées deviennent des idéologies et entraînent des conformismes. Le mouvement féministe était essentiel; il critiquait le rôle réservé aux femmes au Québec, c'est-à-dire la maternité telle qu'elle avait été vécue pendant si longtemps dans les conditions que l'on sait. Évidemment, je partage cette critique. Même à cette époque, j'étais solidaire du mouvement. D'ailleurs, j'ai dit longtemps que je n'aurais pas d'enfants. Il a donc fallu que je fasse une démarche pour admettre que cette position face à la maternité n'était pas la mienne. Ce questionnement se faisait aussi contre certains préjugés idéologiques.

V. et I. : Sans doute est-ce aussi parce qu'à cette époque-là, quand on parlait de la maternité, on associait la naissance à la nature. À partir du moment où beaucoup de femmes ont accès à la culture, cette fonction ne se pense plus de la même façon.

M. L. La rationalité de la femme est une idée que j'ai toujours défendue, bien qu'elle fasse l'objet d'une constante suspicion. Ce n'est pas un cas isolé, il y a de nombreux préjugés contre l'intellectualité en général dans la littérature québécoise. L'entrée de la femme dans la culture passe par le développement de sa raison. La maternité devait selon moi être repensée, il était essentiel d'en renouveler la perception, mais la solution envisagée par plusieurs de ne plus avoir d'enfants m'apparaissait plutôt comme une impasse.

V. et I. : Le thème de la maternité, la figure de la mère traversent vos romans, mais la paternité occupe aussi une place importante, par exemple dans La gloire de Cassiodore, de même que l'enfance et l'adolescence dans Les faux fuyants et Copies conformes, et indirectement dans La démarche du crabe. Il me semble qu'il y a une espèce de dialectique de la famille où alternent les perspectives de la femme, de la mère, du père et des enfants. Comment se conçoit la famille dans votre œuvre?

M. L. : La famille, c'est un cliché de le dire, forme la cellule où se produisent de profondes transformations. Je pense que ce qui m'aura intéressée dans tous mes romans, c'est la question de la transmission. Son importance pour moi est indéniable. En raison de quoi? Je ne le sais pas. Comment se transmet la connaissance ? La transmission - qui n'est pas la même chose que la reproduction - nous définit en tant qu'êtres humains. La culture, le savoir se passent en premier lieu, jusqu'à maintenant, à travers ce qu'on appelle la famille, et plus largement à travers les générations. Mes personnages sont toujours reliés entre générations; je n'ai jusqu'à maintenant pas imaginé de roman qui se construirait autrement.

V. et I. : La question de la transmission du savoir conduit donc à cette famille élargie qu'est la société et, de proche en proche, au politique. La 
démarche du crabe est sans doute le roman qui comporte la plus nette dimension politique, ne serait-ce parce qu'il présente des traces de l'histoire événementielle (mort de Duplessis, les années 1960, l’Expo 67, le discours de De Gaulle, etc.). Sans qu'ils soient des romans historiques au sens strict, n'y a-t-il pas dans vos romans une forme de «cadrage épistémologique »qui renvoie à un climat historique et politique d'ensemble?

M. L : Certainement. Dans La démarche du crabe, c'est quelque chose dont j'étais consciente. Il faut dire que ce texte formait un diptyque dans mon esprit avec le précédent, Copies conformes, que je voyais comme un récit portant vers l'avenir. L'action se passe en Californie et tourne autour d'un personnage québécois à l'étranger, aux prises avec l'anglais, ce qui me semblait être à ce moment-là une question importante d'un point de vue québécois. En mettant en scène un personnage qui régresse, La démarche du crabe présente le cheminement inverse. Le cadre géographique est évidemment le teritoire du Québec et l'histoire se situe chronologiquement autour du deuxième référendum. Je m'intéresse de manière générale assez peu à la politique, mais il était impossible dans ces années-là de ne pas s'interroger. Il m’apparaissait nécessaire de faire un certain deuil du premier référendum. De ce point de vue, La démarche du crabe se voulait un roman sur le deuil, d'abord à l'échelle personnelle, mais qui pouvait avoir rapport aussi, à une autre échelle, avec le deuil du rêve indépendantiste.

V. et I. : La démarche du crabe porte donc sur la perte des illusions, notamment à travers ce personnage central qui est confronté à cette fausse image qu'il a de lui-même.

M. L. : Et cette prise de conscience le mène à la mort. À force de toujours vouloir récupérer, retourner en arrière, obsédé par l'idée de possession, il se retrouve dans une situation d'impasse, un marécage, ce néant, ce trou que représente, en y réfléchissant bien, la source d'un fleuve.

V. et I. : Il est le fantôme de ses propres illusions en quelque sorte: son passé fait corps.

M. L. : C'est juste, il n’a jamais adhéré à la vie, n’a jamais réellement vécu, toute sa vie s'est passée en retrait.

V. et I. : Est-ce que vous voyez ce personnage comme le portrait d'une génération?

M. L. : Peut-être pas d'une génération, mais de bien des gens. On revient à l'idée de transmission. Il faut accepter que certaines choses soient terminées et que, si on se dirige ailleurs, on doit renouveler son regard. C'est bien là que se noue la tragédie dans ce roman: le personnage est incapable de délaisser ses idéaux.

V. et I. : Par contre Robert Dion, dans son article, affirme que la conclusion du récit est étonnamment sereine. Il y a une forme d'acceptation de la part du personnage. 
M. L. : C'est-à-dire qu'il a fait un cheminement, il comprend peut-être mieux dans l'après coup ce qu'il n'a pas su voir auparavant. Je pense que c'est ce que l'on doit faire au Québec; entreprendre ce type de démarche nous mènerait à un nouveau commencement. Pour lui, le cheminement mène à la mort; il était important pour moi d'aller jusque-là, mon intuition me disait que le sens symbolique n'aurait pas été le même s'il avait continué à vivre.

V. et I. : Les personnages de vos textes sont souvent fragiles. Cette fonction que vous attribuiez tantôt au roman de questionner les vérités, les dogmes, les évidences, n'est-elle pas responsable de cette situation? La fragilité des personnages ne provient-elle pas justement de ce qu'ils doutent, s'obligent à être critiques au lieu d'accepter les consensus?

M. L. : Le doute est certainement un thème important pour moi; il me semble former la base de la pensée, de toute réflexion et particulièrement du roman. Le roman est un instrument fin, vaste et riche pour mettre en doute, pour transformer même, d'une certaine manière, la société.

V. et I. : Est-ce qu'on ne pourrait pas dire que dès qu'il est question de filiation et de mémoire, on ne peut pas faire autrement qu'entrevoir le doute ? C'est Valéry qui disait que l'on ne peut tirer de leçon de l'histoire parce que celle-ci donne des exemples de tout. À partir du moment où l'on prend conscience qu'il n'existe pas de progression simple et logique, que tout peut arriver, c'est difficile de ne pas douter.

M. L.: Oui, il me semble que l'on avance en doutant, on pense en doutant, on naît en doutant, on se remet constamment en cause et ce comportement nous fragilise comparativement aux gens qui se complaisent dans l'affirmation. Je serais sans doute incapable de faire un personnage qui ne cesse d'affirmer - bien qu'il y en ait beaucoup autour de nous parce que je ne comprends pas que l'on puisse être entièrement sûr de quelque chose.

V. et I. : De manière générale vos personnages féminins ont su intégrer à leur être, à leur cheminement, des acquis du féminisme. Or, dans vos deux derniers romans, La démarche du crabe et La gloire de Cassiodore, on trouve le constat d'une situation maritale où les attentes ne se sont guère concrétisées, où l'émancipation est de plus en plus difficile. Ces personnages ne sont-ils pas plutôt des figures de femmes sacrifiées?

M. L.: La femme dite «mariée», et plus largement celle qui vit dans un couple depuis longtemps, est en porte-à-faux avec les modèles que lui offre la société. La relation de couple suivie y est jugée peu enviable, et par conséquent les femmes dans cette position se sentent mal; leur choix de vie n'existe pratiquement plus dans la culture actuelle ou à tout le moins ne correspond plus à ce que leur présentent téléromans, films et même romans. Or, dans la réalité sociale, cela existe bel et bien. Mais à 
force de se faire présenter l'union en couple comme une pratique quasi marginale, les femmes qui se trouvent dans cette situation se mettent à douter profondément de leur choix et se demandent si elles ne feraient pas mieux de quitter leur conjoint. Je pense que ce phénomène gruge les couples, et donc encore une fois j'interroge un certain conformisme social. Je connais énormément de femmes qui perçoivent cette manière de vivre comme la plus souhaitable compte tenu qu'elles ont décidé d'avoir des enfants. D'autres aussi, qui ont simplement trouvé du bonheur dans un amour persistant, qui se transforme. Mais le discours ambiant n'a rien de positif pour ce genre de situation. J'ai pointé quelque chose de similaire, je crois, dans La gloire de Cassiodore. Dans ce cas, c'était la figure du professeur dont le rôle est, comme on le sait, de transmettre avec humilité le savoir et la culture, un rôle méprisé par plusieurs, on me le disait même ouvertement dans les entrevues entourant la parution du livre. Je m'interroge sur l'effet et la signification de ce manque d'estime. Je ne comprends pas pourquoi on méprise des gens pour ce genre de raison, vivre en couple, être enseignant. Ce genre de mépris immotivé est un sentiment qui m'irrite profondément. C'est une attitude antiromanesque. Le roman se penche sur tous les êtres humains, il s'y intéresse, au sens fort. Ceci me fait inventer des personnages qui sont en lutte avec l'anti-modèle social. Et c'est la raison pour laquelle je fais apparaître cette personnalité de la «femme mariée», disons, dans La gloire de Cassiodore et dans Copies conformes. Le personnage porte d'ailleurs le même nom dans les deux romans.

V. et I. : Lors de la lecture de La gloire de Cassiodore, certains personnages peuvent à prime abord agacer parce qu'on croit les saisir rapidement. Or ce n'est pas le cas: dans ce roman comme dans les autres, il me semble qu'il y a une attention portée au détail, à la fragilité des êtres, des sujets, qui fait que finalement le personnage que l'on s'imaginait iritant ne l'est plus du tout par la suite.

M. L. C'est bien ce qui m'intéresse dans le roman, de montrer les différentes facettes des êtres, pourquoi ils sont détestables par exemple, contre quoi ils luttent. Je pense que ce n'est pas intéressant d'écrire des romans si on dit la même chose que tout le monde. C'est pour cela que j'essaie de puiser dans ma propre expérience des milieux que je fréquente - qui ne sont pas uniquement littéraires ou intellectuels - , afin d'essayer de voir quelles en sont les idéologies et quels sont les effets de ces dernières dans la vie courante. En ce sens-là c'est politique, et je ne pense pas qu'il y ait beaucoup d'autres arts que le roman qui permettent de faire cela.

V. et I. : Quittons les personnages pour aborder l'espace des romans, qui est presque toujours déterminé par des lieux particuliers: San Francisco dans Copies conformes, le collège dans La gloire de Cassiodore, l'arpentage du territoire dans La démarche du crabe, l'idée de frontière dans Les 
faux fuyants. Est-ce que ces récits sont intrinsèquement liés au lieu où ils se déroulent? Est-ce que, par exemple, San Francisco a réellement déterminé Copies conformes ou c'était plutôt l'idée d'être ailleurs? Quel rôle le lieu joue-t-il dans vos romans?

M. L. : C'est un rôle important. Le lieu n'est pas neutre du tout, c'est un élément de la composition. Écrire une histoire où le lieu n'a pas d'importance me semble sans intérêt. Il faut qu'il y ait un lien entre ce qui va se passer dans l'intrigue et les lieux. Par exemple, dans Copies conformes, San Francisco n'est pas neutre, le roman n'aurait pas pu se passer ailleurs. Mis à part le fait que j'étais en séjour à San Francisco, mon idée était de faire un roman sur le Québécois à l'étranger, comme je l'ai déjà mentionné. Je suis une grande admiratrice du roman Le joueur de Dostoïevski. Copies conformes part un peu de la même idée. Alexis dans Le joueur est à l'étranger, dans un monde sans repères, comme je l'étais un peu en Californie quand j'y suis allée, c'est-à-dire au moment de l'explosion de l'informatique. Le contraste entre la langue maternelle et le bain d'anglais dans lequel est plongé le personnage faisait écho à ce que le Québécois moyen à mon avis ne voulait pas voir - qu'il a toujours encore beaucoup de difficulté à voir - et qui était pourtant là, à nos portes. Il fallait y réfléchir. Le lieu est important aussi dans ce roman en raison de Dashiell Hammett. C'était encore, soit dit en passant, une lutte contre les personnages féminins de Dashiell Hammett que j'entreprenais, même si j'aime beaucoup cet auteur. À chaque fois, le trajet est pensé, nécessaire. Dans La démarche du crabe, il a fallu que j'aille au-delà du cliché que présentait le trajet initial, qui est celui du Michelin, pour le repenser. Bon, pour le collège, c'est évident, c'est le principe du microcosme qui est au cœur du récit, bien plus que la question de l'enseignement que la plupart des commentateurs ont retenue. Ce n'est pas tellement un roman sur l'enseignement, c'est un roman sur une cellule fermée, une micro-société.

V. et I. : Pourtant il est étonnant de voir que bien des gens l'ont perçu comme un récit réaliste. La gloire de Cassiodore n'est-elle pas avant tout une fable sur le savoir, sur les valeurs, sur le monde?

M. L : Les gens lisent souvent au premier degré. Mais dans un collège, les choses ne se passent pas de cette façon, on ne parle pas de cette manière, ce que je veux faire n'est tout de même pas une bête description; tout a été retransformé, et en premier lieu le jargon pédagogique, que j'ai exagéré. Je ne voulais tout de même pas écrire un livre sur mes propres collègues pour amener les gens à chercher les clés. J’ai passé plusieurs heures à m'interroger sur l'art de la fresque avant d'écrire ce roman, et sur ce qu'est un personnage, et un groupe. Beaucoup de lecteurs ont conclu que c'était un roman sur l'enseignement dans les cégeps. Bon, oui, mais présenté tel quel, on rate l'essentiel. C'est une étude de langage. 
V. et I. : Si la littérature devient une histoire de cas, ce n'est plus intéressant. Dans La gloire de Cassiodore, ce n'est pas à ce niveau que cela se joue. L'humour du roman tient justement de ce débordement de la réalité. Cela rejoint ce que vous disiez plus tôt: si l'intrigue avait été située dans un vrai cégep, avec des vrais collègues, le rire aurait été un rire de mépris. Micro-société est, selon moi, le mot clé parce que finalement c'est un roman sur un milieu de travail. Parler d'enseignement, c'est se leurrer; on pourrait regarder n'importe quel groupe de personnes qui se fréquentent, ont des relations entre elles, des douleurs, des plaies qui sont encore ouvertes, etc. Mais, tout de même, que ce soit dans le milieu de l'enseignement signale aussi l'idée de la filiation.

M. L. : On situe souvent l'action d'un récit dans les universités; il y a plusieurs romans américains qui s'y passent, on les apprécie bien en général, on trouve cela drôle. Mais le snobisme intellectuel fait qu'une histoire semblable située cette fois dans un collège, c'est-à-dire dans un ordre d'enseignement plus bas - pas tellement plus bas, juste un peu plus bas -, devient un roman sur l'enseignement, sur la pédagogie, ce n'est soudainement plus intéressant. C'est justement ce que je dis dans ce roman-là. La gloire de Cassiodore traite de la vie intellectuelle au Québec, bien plus que de l'enseignement au collégial. Cette réaction me fâche profondément, sinon je n'aurais pas pris la peine d'écrire tout ce roman.

V. et I. : Dans quelle mesure l'enseignement peut-il être un catalyseur pour la fiction?

M. L. : L'enseignement au collège est un anti-enseignement, un antitravail intellectuel que j'essaie de faire le moins stupidement possible mais qui est quand même anti-littéraire, et donc, oui, cette frustration me pousse à écrire. Une seule journée de cours me met très souvent en colère; le lendemain, j'ai besoin de littérature, c'est le seul moyen pour moi d'y remédier. Je pense qu'il y a beaucoup de personnes qui sont dans ma position. La gloire de Cassiodore en est l'exemple. Après avoir passé ma vie dans un collège à faire de l'anti-littérature alors que je suis une littéraire, à lutter pour que mon cerveau ne soit pas complètement démoli par le ministère de l'Education, j'ai essayé d'en faire un objet littéraire. L'enseignement provoque un effet sur mon écriture, mais ce n'est certainement pas dans mon cas un adjuvant, c'est au contraire un opposant à la vie littéraire. Pour avoir une vie intellectuelle, il faut être hors de ce milieu, et je ne trouve pas cela normal du tout. La situation me semble différente ailleurs, dans les lycées français par exemple. C'est un symptôme grave que d'être incapable d'intégrer la vie intellectuelle à la culture. Le mépris profond que l'on cultive au Québec à l'endroit des intellectuels, des professeurs, des gens qui aiment lire, même si on le dément constamment, est à la source du problème. La preuve en est que 
l'on se fiche de l'enseignement supérieur au Québec, du secteur collégial; même dans les universités on s'en préoccupe peu, à mon avis. Il n'y a pas de solidarité littéraire ou intellectuelle. C'est quelque chose qui me préoccupe et me pousse à écrire, comme un besoin d'oxygène.

V. et I. : Il y a au Québec des milieux littéraires, mais il n'y a pas de réseaux littéraires soutenus par une volonté de créer justement une organisation sociale et culturelle qui tienne compte de la vie intellectuelle, y compris cette activité essentielle qu'est l'enseignement.

M. L. La maîtrise de l'enseignement est méprisée, il me semble que c'est assez évident. Et l'enseignement est divisé en ordres hiérarchiques. Il y a des gens qui parviennent à s'élever à un certain stade et qui ont besoin de regarder en bas en se réjouissant de ne pas y être. C'est humain, mais cela me choque. C'est un vieux thème, que j'ai tenté d'exprimer d'une certaine manière. Si cela n'est pas limpide, ce n'est pas grave, parce que je ne veux pas que mes romans soient limpides.

V. et I. : Lorsqu'on connaît votre intérêt pour Montréal, il est surprenant de voir que cette ville est assez peu présente dans vos romans. Elle est là comme fantasme, comme souvenir, comme image dans Copies conformes; on la traverse un petit peu dans La démarche du crabe, mais c'est à peu près tout. Est-ce que cette absence est volontaire?

M. L. : Lorsque je visitais Montréal à travers le roman dans Promenades littéraires, le projet m'est certainement venu à l'esprit d'écrire à mon tour sur Montréal, mais si je ne l'ai à peu près pas fait, c'est que je ne devais pas juger avoir les moyens pour bien y parvenir. Je suis Montréalaise, mais en même temps qui est Montréalais? J'ai passé ma vie sur la RiveSud, juste de l'autre côté du fleuve, et je continue de penser que le pont Jacques-Cartier est une frontière incroyable. J'ai passé mon enfance à Longueuil. J'enseigne encore dans cette ville, et je peux vous assurer que les élèves de mon entourage sont très peu conscients de l'existence et de la nature de Montréal. Par exemple, je leur ai expliqué demièrement que je devais me rendre au Festival des Films du Monde. On soupçonnait à peine que cela avait lieu à Montréal, de l'autre côté du pont. Je me promets d'aborder ce phénomène dans un roman. Montréal est un espace tellement multiple, tellement riche que je n'arrivais pas à trouver les personnages pour en rendre compte, mais j'ai compris beaucoup de choses sur cette ville à travers l'affaire de L'arpenteur et le navigateur. J'ai vécu, à ce moment-là, une histoire montréalaise. Probablement que maintenant je vais pouvoir écrire un roman montréalais. J'ai compris concrètement cet espace que j'avais regardé à travers les romans, et qui évolue tout le temps. Il y a une certaine honnêteté que je recherche dans mes romans. J'essaie de ne jamais parler de quelque chose que je ne connais pas, et je n'avais pas l'impression encore de connaître suffisamment Montréal. À présent, il me semble que j'en serais capable. 
V. et I. : En quoi ce que vous appelez l'affaire de «'́arpenteur et du navigateur» a-t-elle pu donner lieu à une «histoire montréalaise »? Qu'est-ce qui, dans cette saga autour de la publication de cet essai, a su révéler pour vous un aspect saisissant de cette ville, suffisant pour que vous disiez que vous ne la connaissiez pas suffisamment auparavant?

M. L. : Cela s'explique assez facilement. J'ai toujours vécu le multiculturalisme comme une évidence; c'est ce que j'ai toujours aimé de Montréal. Enfant, j'aimais traverser le pont, et ce fut ma plus grande conquête que d'obtenir la permission de venir à Montréal toute seule. À la fin de mes études, j'ai choisi le collège Marie de France parce que j'étais fatiguée de l'enseignement des sœurs, mais surtout parce que c'était la France à Montréal et que la France exerçait une fascination sur moi. Ensuite, j'ai choisi l'Université McGill avant de poursuivre mes études à l'Université de Montréal. J'ai beaucoup aimé McGill, être dans une langue étrangère; toute cette faune multiethnique rassemblée dans un même lieu me réjouissait. J'ai dû finalement quitter cette université parce qu'il s'avérait de plus en plus difficile de suivre les cours de linguistique en anglais pour la francophone que j'étais. Aujourd'hui, certains de mes meilleurs amis appartiennent à la communauté juive, mais aussi italienne, hispanophone, etc. Mes enfants ont été élevés dans un milieu tellement multiculturel que c'est tout à fait stimulant de voir la diversité des noms qui apparaissent sur l'afficheur téléphonique; c'est un poème en soi. Par conséquent, j'ai été très surprise de ce qui m’est arrivé. Les réactions qu'a suscitées la sortie de ma conférence m’ont dévoilé l'illusion qu'avait été jusque-là pour moi cette fête montréalaise. J'ai quand même beaucoup voyagé, vu plusieurs grandes villes et il n'y en a que très peu que j'aime autant que Montréal. Je ne soupçonnais pas, avant cet événement, ce malentendu incroyable - qui à son origine faisait écho à un problème bien plus large - l'existence de ce malaise au sein de l'ensemble de la communauté entre ceux que je vais appeler les Canadiens français, faute d'un autre mot pour désigner les Québécois d'origine principalement francophone, et les autres. J'ai compris que derrière l'image simplifiée d'un multiculturalisme sans anicroches que nous renvoient les nombreux festivals de Montréal, la réalité était infiniment plus complexe. J'ajouterais que ce mirage réconfortant est celui d'une certaine bourgeoisie intellectuelle à laquelle j'appartiens, où le fait que l'on fréquente toutes les cultures, où que l'on soit multilingue, va de soi, ce qui n'est pas vrai du tout. Je n'ai pas pensé une seconde que l'on pourrait recevoir mon discours différemment, et j'ai appris énormément de cette mésaventure. En ce sens, l'épisode m'a fait un peu mieux comprendre le tissu profond de Montréal. Une ville n'est pas simplement un espace de fête; c'est aussi un espace polémique. Cela m'a permis d'imaginer Montréal comme un lieu dramatique ou, en tout cas, un lieu où il pourrait y avoir un récit possible, ne serait-ce que celui de cette illusion dans laquelle j'étais par 
rapport à cette paix et à cette amitié qui me semblaient caractéristiques de Montréal telle que je la connaissais.

V. et I. : En tout cas, l'affaire a révélé effectivement un malentendu parce qu'on n'avait pas connu depuis fort longtemps une polémique de cette ampleur, et bien vite les interventions se sont mises à ne porter qu'indirectement sur L'arpenteur et le navigateur pour aborder des sujets qui s'en éloignaient.

M. L.: Oui, il y a quelque chose derrière dont je n'avais pas soupçonné l'ampleur, certainement pas, même au sein du milieu des écrivains. Ce débordement auquel le débat a donné lieu montre un problème qui existe et dont la complexité vaut la peine d'être inventoriée. C'est en grande partie, selon moi, un problème de communication : à qui s'adresse-t-on? Qui reçoit le discours? Quel est le contenu de ce discours? De quelle façon le contenu du discours varie-t-il selon le récepteur? Cet épisode a complexifié mon appartenance à Montréal.

V. et I. : Revenons à des questions plus directement littéraires. Il y a évidemment toutes sortes de résonances littéraires dans vos romans, Dashiell Hammett bien sûr, mais aussi la structure du road book dans Les faux fuyants. En fait, les exemples sont nombreux. Quelles ont été vos grandes influences littéraires, culturelles et intellectuelles?

M. L. : C'est difficile pour moi de tout nommer. Qu'est-ce qui m'a influencée ? Eh bien, Roland Barthes, bien sûr, tout le milieu français à Paris dans les années 1970: Bourdieu, Lacan, Foucault. J'allais à tous ces séminaires, c'était passionnant. Mais en même temps, cela ne me menait nulle part. Par ailleurs, je me suis laissée beaucoup influencer, si l'on veut, par la théorie du roman, qui continue encore de me passionner. Les cours gravitant autour de l'art du roman sont les seuls que j'aie pu donner, parfois, au collège qui m'ont intéressée, notamment parce qu'ils s'adressaient à des élèves faisant partie de ce qu'on appelait le programme international, que l'on s'est empressé d'abolir. Une autre source d'inspiration sont les grands romans, ceux du vingtième siècle, que j'essaie de lire en priorité, ceux des autres cultures et des autres langues. Mais une influence directe de tel ou tel artiste, je ne pourrais pas en nommer.

V. et I. : Vous avez quand même parlé du Joueur de Dostoïevski.

M. L.: Oui, c'est un grand roman, que j'ai lu et relu. J'ai tendance à relire des romans plusieurs fois. Pendant une assez longue période, j’ai probablement été très marquée par Thomas Bernhard parce que je l'ai lu et relu. Le roman américain a aussi sûrement laissé quelques traces chez moi : me viennent tout de suite en tête Philip Roth, William Gaddis et Don Delillo. J'aime particulièrement Virginia Woolf, j'admire sa prose. Je m'intéresse aux écritures en prose; je cherche comment les auteurs arrivent à faire passer des critiques ou des visions sociales à travers une prose fluide, concrète, même terre à terre. Quand j'écris, je me réfère 
aussi à d'autres arts. Comme je l'ai dit tantôt au sujet de La gloire de Cassiodore, j'avais lors de sa rédaction l'impression de peindre une fresque. J'ai donc regardé, dans l'histoire de la peinture, de quoi retournait la composition d'une grande fresque, comment un personnage appartient à un groupe, comment son visage, en Occident, est individualisé alors qu'il ne l'est pas nécessairement dans une autre culture. Qu'est-ce qu'un portrait de groupe? Je suis allée au Mexique et j'ai été très impressionnée par Diego Rivera. À l'amorce d'un roman, je me vois comme un peintre, j'essaie de ne pas fignoler un détail avant que ne se dessine en moi l'idée générale. De même, la musique est toujours présente lorsque j'écris; le mouvement et le rythme d'une phrase en proviennent régulièrement. En composant La gloire de Cassiodore, j'ai été attentive aux sonorités. Il y a des gens qui s'en sont aperçus en disant que c'était très cacophonique. C'était probablement ma façon de transmettre un sentiment d'enfermement à travers la sonorité même de la langue. Le jazz m'intéresse, j’aimerais en percer le secret, mais je n'y arrive pas. Ma formation est très classique, je suis hyper-française de formation, par la philosophie, par la langue, par ce que j'enseigne, qui est restreint à la langue française le plus souvent. J'essaie donc de me décloisonner, de passer à d'autres langues. J'essaie toujours d'apprendre une autre langue en espérant pouvoir renouveler par son entremise ma perception de manière générale, et mon rapport à la langue française. J'apprends l'espagnol en ce moment. La photo m'intéresse aussi, je me demande souvent ce qu'est un regard. Un romancier doit aussi être photographe, c'est-à-dire être capable d'avoir un regard actif. C'est très difficile d'être un bon photographe. Le cinéma m'apporte aussi énormément, c'est un art dont les techniques sont intimement liées au roman. Il y a beaucoup de choses qui m'influencent. L'art du jardin, par exemple, me semble intéressant pour un romancier. Il me semble que l'énumération pourrait se continuer indéfiniment.

V. et I. : Il y a des choses que le roman ne laisse pas voir, c'est peut-être une des différences avec la photographie ou le cinéma. Il peut y avoir du non-dit dans le cinéma, mais si on montre une pomme par exemple, on va percevoir immédiatement sa couleur tandis que dans un roman si on dit une pomme, le lecteur doit imaginer sa couleur, les détails.

M. L. : Absolument, il faut tout dire. L'art du cinéma est écrasant par rapport à l'art du roman parce qu'on entend et on voit, alors que dans le roman, on peut entendre les conversations mais pas les bruits, qui sont très difficiles à rendre. Tout ce qui est perception sensorielle, le romancier est obligé de l'écrire, et la réussite n'est pas garantie. Le cinéma est plus performant à ce niveau.

V. et I. : Par contre, la liberté est plus difficile à atteindre au cinéma.

M. L. : Ah oui, bien sûr, c'est horrible. Je serais incapable de faire du cinéma, ne serait-ce que parce qu'il faut être un homme ou une femme 
d'affaires. J'aime le peu de moyens que demande l'écriture d'un roman, un quasi rien, je veux dire, crayon, papier, même ordinateur, cela ne coûte rien. C'est formidable.

V. et I. : Votre premier roman, La cohorte fictive, est très fragmenté; le second l'est aussi passablement. Par la suite, l'écriture devient plus resserrée. Dans La gloire de Cassiodore, la multiplicité des voix et la cacophonie qu'engendre leur entrecroisement n'empêchent pas qu'elles soient très liées. Est-ce un hasard ou un choix esthétique, ce retour vers une forme textuelle plus traditionnelle après le premier roman?

M. L. : Lorsque j'écrivais La cohorte fictive, l'influence de Barthes, l'apologiste du fragment, était encore très forte. Je n'aurais pas osé écrire un texte non fragmenté à cette époque. Par la suite, je me suis rendu compte que la force d'attraction qu'exerçait sur moi un roman était toujours redevable à un fil narratif suivi. Contrairement à ce que la logique voudrait, on commençait à mon époque par l'anti-roman, un peu comme quelqu'un qui débuterait par l'abstraction en peinture et qui n'aurait pas au préalable appris à dessiner ou à représenter le réel. J'ai débuté avec l'anti-roman, le nouveau roman, et l'hostilité envers le genre romanesque, pour ensuite apprendre ce qu'était vraiment le roman traditionnel. C'est, je crois, la raison qui explique que mes romans sont devenus en effet de plus en plus liés, avec une narration plus linéaire. Ne pouvant faire autrement, j’ai procédé à l'envers.

V. et I. : Par leur construction, les personnages de vos romans provoquent généralement chez le lecteur un sentiment fort d'identification. Les faux fuyants ne représente-t-il pas aussi à ce chapitre une exception? On y retrouve toutes sortes d'effets de distanciation qui en font un roman différent, malgré la présence de vos thèmes de prédilection.

M. L. : Les faux fuyants traite de l'errance, du nomadisme. Les personnages mis en scène n'ont pas de point d'ancrage, aucune racine, aucune famille, ils sont jetés dans l'existence. Tout être humain fait un jour cette expérience qui va à l'encontre d'une transmission, celle d'une absence d'abri, de tout abri. C'est difficile pour moi de mesurer l'effet de lecture, il faut admettre le fossé existant entre l'écrit et la lecture qui en est faite. Je ne déteste pas m'identifier à un personnage dans un roman, ce penchant explique peut-être que mon écriture produise cet effet-là. Si Les faux fuyants s'en écarte, c'est qu'il est davantage un roman d'observation qu'un roman introspectif, bien qu'il parte d'une certaine expérience personnelle. Une chose est certaine, plus j'avançais dans cet art du roman, plus le personnage devenait important. $\mathrm{Si}$ on se rappelle l'époque de Barthes, le personnage était un être de papier, c'était une voix. Il était presque disparu, comme tous les éléments du langage romanesque. Jeune romancière, j'ai entretenu pendant un petit bout de temps une correspondance avec Gérard Bessette, que j'admirais, que je continue d'admirer 
beaucoup. Je lui avais demandé s'il fallait encore écrire avec des personnages. Il m'avait répondu en trois mots: «personnage pas mort». Cette réponse on ne peut plus claire m'a fait faire un bout de chemin, et plus je continue à avancer, plus je vais vers les personnages. Mon dernier livre, La gloire de Cassiodore, n'est fait pour l'essentiel que des relations entre des personnages. Lors de l'écriture, je sais que je touche à l'essence d'un personnage à partir du moment où il devient quelqu'un que je ne connais pas. La conception d'un personnage nécessite une traversée vers l'autre, pour prendre un mot à la mode; mais c'est quand même cela, il faut parvenir à imaginer l'autre-que-soi à partir de soi, pour atteindre, peut-être, l'humain.

V. et I.: Au Québec, on n'a jamais eu de maitre à penser comme le fut par exemple Roland Barthes en France. Est-ce une bonne chose? D'un côté, cette figure peut être entrevue comme un fardeau pour ses épigones, de l'autre, elle peut apparaître comme la possibilité d'une filiation entre penseurs. On n'apprendra rien à personne en disant qu'il faut des lois pour qu'on puisse les contourner, qu'il faut des statues pour qu'on puisse les abattre. Le paysage intellectuel québécois n'a jamais présenté une telle dynamique. N'est-ce pas un problème, cette absence de grands maîtres intellectuels auxquels s'opposer?

M. L.: Peut-être que l'on préfère prendre nos maîtres ailleurs, on n'a pas à s'opposer à eux. À l'époque dont je parle, la France était très influente partout, non seulement ici. Les gens au Québec répétaient souvent un certain discours qui provenait de France, et ce comportement ne date pas d'hier. Mais je ne peux pas dire que Roland Barthes a été un maître, ce n'était pas quelqu'un qui voulait s'imposer. Je l'admirais, lui et son œuvre, son autonomie et son indépendance intellectuelle. C'est cette figure intellectuelle d'homme libre qui a été un modèle pour moi. Il m’a beaucoup appris en ce sens-là. Il m’apparaît évident que si on méprise la vie intellectuelle comme c'est le cas au Québec, le maître n'est pas une figure qui compte. On ne s'oppose pas au maître, on le méprise parce qu'on est plus riche que lui ou parce qu'on ne comprend pas pourquoi il a décidé d'enseigner. On me pose assez souvent la question: «Pourquoi êtes-vous professeur de français?», avec une certaine pitié dans la voix. Cet exemple montre bien la raison, à une certaine échelle, de cette absence de maître: la vie intellectuelle récolte bien peu d'intérêt au Québec. Je pense sincèrement que la vie intellectuelle mérite d'être racontée, elle est intéressante; la progression intellectuelle est un récit en soi. Malheureusement, ce n'est pas vu comme une matière vivante faisant partie du social.

V. et I. : Vous avez participé activement à la vie littéraire à partir de la fin des années 1970, notamment au développement de la revue Spirale et de La Nouvelle Barre du jour. Pourtant, dans ce deuxième cas, tout en étant liée à ce qu'on appelait la nouvelle écriture, vous n'y avez que très peu 
publié. Y avait-il une raison? Quelle était votre situation par rapport à ce mouvement?

M. L. : Je me vois comme une marginale vis-à-vis de ce courant qu'était la nouvelle écriture. J'y connaissais forcément beaucoup de gens, étant donné la petitesse du milieu littéraire, mais je me percevais comme une romancière et comme une intellectuelle; je ne me sentais pas du tout poète. Je ne suis malheureusement pas lyrique, bien que je sois amenée depuis longtemps à aller vers la poésie, pour tenter de la comprendre, ce qui va peut-être un jour donner des résultats. Je lis beaucoup de poésie moderne, mais je n'en ai pas parlé parce que cela n'influence pas directement mon travail de romancière. Je suis allée vers le roman dans ce qu'il a de plus traditionnel et de plus universel, c'est ce qui explique en partie pourquoi je ne coïncidais pas avec l'esthétique de la nouvelle écriture de La Nouvelle Barre du jour. Je ne comprenais pas et je ne comprends toujours pas certaines démarches de gens que je connais de près ou de loin, mais je les respecte entièrement. Tout en étant à l'écart du mouvement, je revendiquais tout de même avec eux l'idée de modernité, et bien d'autres qu'ils défendaient. À travers la revue Spirale, on avait trouvé un terrain fort intéressant de collaboration et de grande ouverture. Justement, il y avait des gens qui faisaient partie plus précisément de cette nouvelle écriture mais qui étaient ouverts à ce que je pouvais faire, et l'inverse était tout aussi vrai. Au cours des premières années de Spirale, les milieux n'étaient pas aussi cloisonnés qu'ils le sont maintenant, entre l'université et le collège. Il y avait beaucoup d'intellectuels des collèges qui participaient à Spirale. J'ai collaboré au Devoir aussi. J'essaie de garder une écriture qui n'est pas de création, mais d'intervention, parce que comme je l'ai déjà dit, ce qui m'influence le plus, c'est mon milieu. Je reste en contact avec ce milieu littéraire par le biais de tous ces colloques qui ont lieu et auxquels je participe.

V. et I. : Une question un peu plus vaste pour terminer: comment voyezvous l'instance critique aujourd'hui au Québec, l'institution littéraire en général, le monde de l'édition, de la critique? Est-ce qu'il y a des particularités ici, positives ou négatives?

M. L. : La critique a beaucoup changé depuis le moment où j'ai commencé à publier, c'est devenu davantage du reportage que de la critique littéraire à proprement parler. Je pense quand même qu'il ne faut pas trop se plaindre, on a quand même la chance au Québec de pouvoir être publié par de bonnes maisons d'édition, avec un travail d'édition qui est vraiment remarquable. Il m'arrive assez souvent d'ouvrir des livres d'éditeurs français où il y a plus de fautes que dans les livres des bonnes maisons d'édition québécoises. Quelqu'un qui est écrivain à New York ou à Paris a sûrement beaucoup de difficulté à se faire publier. Il est certain que nous écrivons pour un petit public, mais cela a fini par m'être assez 
indifférent, bien qu'il y ait beaucoup de pression dans le milieu vers l'internationalisation et les débouchés vers d'autres marchés, etc. Le marché, en tant qu'écrivain, n'est pas mon problème. Je me suis toujours sentie en marge de ces questions. Il me semble que c'est l'une des facettes du métier d'écrivain que d'accepter une grande solitude; c'est le garant indispensable pour faire une œuvre qui ne soit pas comme les autres, pour avoir sa propre voix. Assumer cette solitude n'est pas chose facile. Écrire, c'est être tout petit et invisible, dans un monde très riche. De grands livres sortent tous les jours et cela mène, je pense, à une grande humilité, bien que ce n'est pas un mot que j'aime utiliser puisqu'il connote un certain défaitisme. Il faut voir l'immensité des choix qui s'offrent à nous en ce moment, on a accès à tout, aux œuvres de tous les pays et de toutes les époques, alors que c'est plus que ce que l'on peut consommer et a fortiori fréquenter dans une seule vie. 